\title{
Risk Elements in Drinking Water of Koya City
}

\author{
Lana Omer Ahmed ${ }^{1, *(\mathbb{D})}$, Maan Al-Arif ${ }^{1}(\mathbb{D})$, Faten Chaqmaqchee ${ }^{2(\mathbb{D}}$, Rebaz Omer $^{3}{ }^{(\mathbb{C}}$ \\ 1 Department of Physics, Faculty of Science \& Health, Koya University, Koya KOY45, Kurdistan Region - F.R. Iraq; \\ lana.omer@koyauniversity.org (L.O.A.), faten.chaqmaqchee@koyauniversity.org (F.C.); \\ 2 Department of Medical Laboratory, Knowledge University, PO Box 074016 Daratoo st. 123, Kirkuk Road, Kurdistan \\ Region, Iraq; maan.safa@koyauniversity.org (M.A.A.); \\ 3 Department of Chemistry, Faculty of Science \& Health, Koya University, Koya KOY45, Kurdistan Region - F.R. Iraq; \\ rebaz.anwar@koyauniversity.org (R.O.); \\ * Correspondence: lana.omer@koyauniversity.org (L.O.A.);
}

Received: 23.06.2021; Revised: 27.07.2021; Accepted: 30.07.2021; Published: 5.09.2021

\begin{abstract}
This study is based on an analysis of drinking water at Koya city, Kurdistan-Iraq. Since the source of tap water comes either from Dokan Lake or from groundwater resources. Therefore, ten samples from houses tab water of Koya city and ten samples from well-known brands of bottled water were collected. Concentrations of the metals in such water samples were analyzed by X-ray fluorescence and compared with WHO water quality standards for minerals. The experimental results show that most elements detected in this study were within the guidelines given by WHO for drinking water, while four elements of $\mathrm{Ca}, \mathrm{Al}, \mathrm{Mg}$, and $\mathrm{Cr}$ were identified as risky elements in developing diseases since their level exceed the WHO standard levels. Positive relationships between risky elements and some serious diseases were discussed. Statistical work shows that elements; $\mathrm{Ca}, \mathrm{Al}$, and Cr have a high risk while $\mathrm{Mg}$ has a low risk.
\end{abstract}

Keywords: drinking water; water analysis; water quality; water elements; XRF.

(C) 2021 by the authors. This article is an open-access article distributed under the terms and conditions of the Creative Commons Attribution (CC BY) license (https://creativecommons.org/licenses/by/4.0/).

\section{Introduction}

On average, individuals need about 2-3 liters of water per day depending on the nature of the climate and physical activity, and the culture of the community [1]. Bottled water consumption has been growing steadily worldwide during the past three decades [2]. It is considered as one of the fastest-growing and most vital of all the food and beverage factories. The growing demand for bottled water in Iraq could be restricted to different reasons such as water scarcity, change in the characteristics of drinking water quality, and foreign communities found after the occupation of Iraq [3]. In addition to the weak confidence of the consumer in the validity of drinking water supplied from government projects [4]. This rapid and untracked increase in bottled water prompts concerns about bottled water quality in the market. Legally bottled waters in Iraq should comply with the Iraqi and international standards to be licensed by General Directorate for Industrial Development.

There is considerable debate among scientists about the effects of minerals in drinking water. Heavy metals in water have become of particular interest in recent decades within the framework of environmental investigation $[5,6]$, where the metal content can be detected by highly sensitive analytical procedures with high precision. In general, the trace constituents are not always evaluated in production. However, metallic elements such as $\mathrm{Al}, \mathrm{Cr}, \mathrm{Fe}, \mathrm{Cu}, \mathrm{Zr}$, and $\mathrm{Zn}$, have important performances in several cellular functions. Under normal conditions, these 
elements are in balance in the body. An excessive accumulation can result in degeneration or death of some tissues, compromising all the health of the body. The presence of radioactive elements such as uranium in drinking water may lead to harmful biological effects in humans; for example, the chemical toxicity of natural uranium is a major hazard to the kidney [7-9].

In the Kurdistan region of Iraq, the quality of natural drinking water is generally fluctuated depending on the underground water resource property. The tap water source, especially in KOYA city, comes either from Dokan Lake or underground wells. The number of studies on drinking water quality in Erbil and other Kurdistan cities accomplished mainly using physical, chemical, and biological analysis [10-13]. As a result, water in some sites has a high percentage of sodium, sulfate, and calcium, while in other sites, it showed evidence of pollution. None of these studies reflect exactly the property of drinking water at Koya city, which is located in the southeast of Erbil and is surrounded by the Bawaji, Sari Kosar, and Haibat Sultan mountains. The lack of information about drinking water quality in Koya City encourages us to do this study.

This study has determined the level of elements in drinking water compared with the international standards and determine its impact on health. This task is done using the $\mathrm{x}$-ray fluorescence technique (XRF) available at the department of physics of Koya University.

\section{Materials and Methods}

$\mathrm{X}$-ray fluorescence (XRF) is a laboratory-based technique that uses the emission of characteristic fluorescent X-rays from a material that has been excited by being bombarded with high-energy X-rays. The phenomenon is widely used for elemental analysis, particularly in investigating metals, building materials, and for research in geochemistry, forensic science, and art objects [14]. Twenty drinking water samples from Koya city were analyzed using this technique. Ten of them were collected from the most commonly consumed bottled drinking water. The other ten samples were collected from various sources of tap water. A water sample of $8 \mathrm{~mL}$ was poured into the sample cell supplied with a base of $4 \mu \mathrm{m}$ Prolene film. The sample cell was placed into an oven at $45^{\circ} \mathrm{C}$ for 3 days to evaporate water and allow minerals to precipitate as a uniform thin layer over the Prolene thin film. All the samples were analyzed using the Energy Dispersive X-ray Florescence Rigaku NEX CG machine available at the department of physics of KOYA University. An energy-dispersive detector was used to determine the composition of the elements. The target material of the X-ray tube is palladium (Pd). The (Pd) tube has high excitation efficiency for heavy and light elements with its characteristic X-rays of the $\mathrm{K}$ and L-series. There are four secondary targets inside the machine ( $\mathrm{Mo}, \mathrm{Cu}, \mathrm{Al}$, and RX9). Each of them can detect a group of elements. The secondary target is selected automatically according to components contained in the sample or the elements to be analyzed. All water samples were processed and analyzed, and the precipitated mineral masses are shown in Table 1.

In this work, the statistical parameters of minimum, maximum, mean, SD, and percentage coefficient of variation $(\% \mathrm{CV})$ were determined after the data distribution passed the normality test $(\mathrm{P}<0.05)$ and only for the data containing five or more values. Otherwise, the statistical parameters were labeled as not applicable (NA). The \%CV value greater than $100 \%$ represents a highly variable distribution around the mean, i.e., SD exceeds the mean. 
Table 1. Water sources and precipitated mineral mass in $8 \mathrm{ml}$ of the samples.

\begin{tabular}{l|l|l|l|l} 
Sample number & Bottle water & Precipitate mass $(\mathbf{m g})$ & Tap Water & Precipitate mass $(\mathbf{m g})$ \\
\hline 1 & Aqua & 1.2 & Koya-Shahidan & 9.8 \\
\hline 2 & Ma'een & 1.0 & Koya-Shaugeraua & 2.1 \\
\hline 3 & Lava & 1.2 & Koya-Bafriqandil & 2.0 \\
\hline 4 & Nil & 1.9 & Koya-Sairan City & 1.6 \\
\hline 5 & Life & 1.4 & Koya-Azadi & 1.5 \\
\hline 6 & Jin & 1.6 & Koya-Haibatsultan & 2.9 \\
\hline 7 & Mina & 0.6 & Koya-University & 5.8 \\
\hline 8 & Noor hayat & 1.4 & Taqtaq-Qalata & 1.9 \\
\hline 9 & Jiyan & 1.3 & Taqtaq-xalxalan & 3.8 \\
\hline 10 & Alwaha & 1.7 & Koya-Nali & 1.8
\end{tabular}

\section{Results and Discussion}

Tables 2 and 3 show the analysis results of different kinds of bottled water samples and their statistical parameters, respectively. Some elements appear with a high concentration in all bottled water samples, such as $\mathrm{Ca}, \mathrm{Na}, \mathrm{Mg}, \mathrm{S}, \mathrm{Cl}, \mathrm{Zr}, \mathrm{Si}, \mathrm{Al}, \mathrm{K}, \mathrm{Fe}$, and $\mathrm{Zn}$.

Table 2. Percent relative mass concentration of elements contained in bottled water samples.

\begin{tabular}{|c|c|c|c|c|c|c|c|c|c|c|}
\hline$\stackrel{9}{3}$ & \multicolumn{10}{|c|}{ Bottled water samples } \\
\hline$\%$ & 1 & 2 & 3 & 4 & 5 & 6 & 7 & 8 & 9 & 10 \\
\hline $\mathrm{Ca}$ & ---- & 3.46 & 84.2 & 75.6 & 84.3 & 10.7 & 44.9 & ---- & 43.2 & 1.58 \\
\hline $\mathrm{S}$ & 0.569 & 6.87 & 0.622 & 4.46 & 6.14 & 10.8 & 6.68 & 31.6 & 5.82 & 39.7 \\
\hline $\mathrm{Cl}$ & 0.712 & 11.8 & 0.797 & 3.15 & 0.919 & 9.38 & 4.40 & 30.4 & 19.7 & 5.41 \\
\hline $\mathrm{Mg}$ & 23.6 & 8.04 & 8.89 & 8.37 & 7.15 & 27.0 & 20.4 & 4.22 & 15.3 & 31.8 \\
\hline $\mathrm{Si}$ & 2.18 & 8.24 & 1.40 & 1.44 & 0.985 & 10.4 & 8.81 & 5.78 & 11.7 & 4.58 \\
\hline $\mathrm{Zr}$ & 58.1 & 0.736 & 0.140 & --- & 0.231 & 0.650 & 10.0 & 0.0122 & 2.38 & 0.941 \\
\hline $\mathrm{Al}$ & --- & 0.568 & 0.0515 & 0.123 & 0.202 & 0.501 & 2.99 & --- & 0.532 & 1.11 \\
\hline $\mathrm{K}$ & --- & 0.342 & --- & --- & --- & 1.33 & 0.664 & --- & 0.585 & 0.284 \\
\hline $\mathrm{Fe}$ & 0.0181 & 0.0888 & 0.0098 & --- & 0.0148 & 0.0419 & 0.475 & --- & 0.224 & 0.0691 \\
\hline $\mathrm{Zn}$ & 0.0005 & 0.0138 & 0.0012 & --- & 0.0008 & 0.0020 & 0.0588 & --- & 0.0754 & 0.0031 \\
\hline Sc & --- & 0.0213 & --- & --- & --- & 0.0182 & --- & --- & 0.188 & 0.0060 \\
\hline $\mathrm{Sr}$ & 0.0015 & 0.0032 & 0.0012 & --- & 0.0048 & 0.0060 & 0.0253 & 0.0002 & 0.0168 & 0.0014 \\
\hline $\mathrm{Ti}$ & --- & 0.0128 & --- & --- & --- & 0.0133 & 0.103 & --- & 0.0312 & 0.0074 \\
\hline $\mathrm{Br}$ & 0.0003 & 0.0008 & 0.0002 & --- & 0.0002 & 0.0013 & --- & --- & 0.0087 & 0.0004 \\
\hline $\mathrm{Cr}$ & --- & 0.0041 & $\begin{array}{l}-- \\
\end{array}$ & --- & --- & 0.0059 & 0.0484 & --- & 0.0134 & 0.0040 \\
\hline $\mathrm{Tm}$ & --- & --- & --- & --- & --- & --- & --- & --- & --- & 0.0007 \\
\hline $\mathrm{Re}$ & 0.0004 & 0.0041 & --- & --- & --- & 0.0013 & 0.0381 & --- & 0.0138 & 0.0019 \\
\hline $\mathrm{Fr}$ & 0.0002 & 0.0012 & --- & --- & --- & 0.0005 & --- & --- & 0.0027 & 0.0008 \\
\hline $\mathrm{Tb}$ & --- & 0.0037 & --- & --- & --- & 0.0062 & 0.0778 & --- & 0.0410 & 0.0039 \\
\hline $\mathrm{Au}$ & 0.0004 & --- & --- & --- & --- & --- & --- & --- & --- & --- \\
\hline $\mathrm{Pa}$ & --- & --- & --- & --- & --- & --- & --- & --- & 0.0656 & --- \\
\hline Dy & 0.0025 & 0.0111 & --- & --- & --- & 0.0066 & 0.110 & --- & 0.0287 & 0.0020 \\
\hline $\mathrm{Mn}$ & --- & 0.0069 & --- & --- & --- & --- & 0.0806 & --- & --- & --- \\
\hline $\mathrm{Cu}$ & 0.0004 & 0.0037 & --- & --- & --- & 0.0011 & 0.0224 & --- & --- & 0.0021 \\
\hline $\mathrm{Ta}$ & --- & 0.0044 & --- & --- & --- & 0.0020 & --- & --- & --- & --- \\
\hline $\mathrm{Na}$ & 14.8 & 59.8 & 3.88 & 6.88 & --- & 29.2 & --- & 28.0 & --- & 14.5 \\
\hline Hf & 0.0006 & --- & --- & --- & --- & --- & 0.0673 & --- & 0.0342 & \\
\hline $\mathrm{W}$ & --- & --- & --- & --- & 0.0006 & --- & --- & --- & --- & 0.0034 \\
\hline $\mathrm{Rb}$ & --- & --- & ----- & --- & --- & --- & ----- & --- & --- & --- \\
\hline $\mathrm{Pt}$ & --- & --- & --- & ---- & --- & --- & --- & --- & --- & --- \\
\hline $\mathrm{Sn}$ & --- & --- & --- & --- & $\begin{array}{l}---- \\
\end{array}$ & --- & --- & --- & --- & --- \\
\hline $\mathrm{Pb}$ & --- & --- & --- & --- & ----- & --- & --- & --- & --- & --- \\
\hline $\mathrm{Er}$ & 0.0013 & 0.0087 & --- & --- & --- & --- & --- & --- & --- & --- \\
\hline $\mathrm{Ni}$ & $\begin{array}{l}-- \\
\end{array}$ & --- & $\begin{array}{l}-- \\
\end{array}$ & $\begin{array}{l}-- \\
\end{array}$ & $\begin{array}{l}-- \\
\end{array}$ & 0.0013 & $\begin{array}{l}-- \\
\end{array}$ & --- & $\begin{array}{l}-- \\
\end{array}$ & --- \\
\hline $\mathrm{V}$ & --- & 0.0030 & --- & --- & --- & 0.0102 & --- & --- & --- & --- \\
\hline Po & --- & --- & --- & --- & --- & --- & ----- & --- & --- & --- \\
\hline $\mathrm{Eu}$ & 0.0046 & --- & --- & --- & --- & --- & 0.0365 & --- & --- & --- \\
\hline $\mathrm{Yb}$ & --- & --- & --- & --- & --- & --- & --- & ------ & --- & ----- \\
\hline At & --- & --- & --- & --- & --- & --- & --- & --- & 0.0050 & --- \\
\hline Ir & --- & --- & --- & --- & --- & --- & --- & --- & ----- & --- \\
\hline $\mathrm{Bi}$ & --- & --- & --- & --- & --- & --- & --- & --- & ----- & --- \\
\hline
\end{tabular}


The element of $\mathrm{Ca}$ was found with concentration ranging between $0.0 \%$ to $84.3 \%$, a mean value of $43.49 \pm 35.44 \%$ and $\% \mathrm{CV}$ equals to $81 \%$, Na found with concentration ranging between $0.0 \%$ to $59.8 \%$, a mean value of $22.44 \pm 19.06 \%$ and $\% \mathrm{CV}$ equals to $81 \%, \mathrm{Mg}$ found with concentration between $4.22 \%$ to $31.8 \%$, a mean value of $15.48 \pm 9.6 \%$ and $\% \mathrm{CV}$ equals to $62 \%, \mathrm{~S}$ found with concentration between $0.569 \%$ to $39.7 \%$, mean value of $11.33 \pm 13.3$ and $\% \mathrm{CV}$ equals to $117 \%, \mathrm{Cl}$ found with concentration ranging from $0.712 \%$ to $30.4 \%$, a mean value of $8.67 \pm 9.71 \%$ and $\% \mathrm{CV}$ equals to $112 \%, \mathrm{Zr}$ with concentration between $0.0 \%$ to $58.1 \%$, a mean value of $8.13 \pm 19.0 \%$ and $\% \mathrm{CV}$ equals to $234 \%$, Si found with concentration ranging between $0.985 \%$ to $10.4 \%$, a mean value of $5.55 \pm 4.03 \%$ and $\% \mathrm{CV}$ equal to $73 \%, \mathrm{Al}$ found with concentration ranging between $0.0 \%$ to $2.99 \%$, a mean value of $0.76 \pm 0.96 \%$ and $\% \mathrm{CV}$ equals to $127 \%$; $\mathrm{K}$ found with range between $0.0 \%$ to $1.33 \%$, a mean value of $0.64 \pm 0.42 \%$ and $\% \mathrm{CV}$ equal to $65 \%$, Fe found with concentration ranging between $0.0 \%$ to $0.475 \%$, a mean value of $0.12 \pm 0.16 \%$ and $\% \mathrm{CV}$ equals to $136 \%$ and $\mathrm{Zn}$ found with concentration ranging between $0.0 \%$ to $0.0754 \%$, a mean value of $0.02 \pm 0.03 \%$ and $\% \mathrm{CV}$ equal to $155 \%$.

Table 3. Statistical parameters for elements contained in bottled water samples.

\begin{tabular}{|c|c|c|c|}
\hline$\%$ Elements & $\%$ Mean & SD & $\% \mathrm{CV}$ \\
\hline $\mathrm{Ca}$ & 43.49 & 35.44 & 81 \\
\hline $\mathrm{S}$ & 11.33 & 13.30 & 117 \\
\hline $\mathrm{Cl}$ & 8.67 & 9.71 & 112 \\
\hline $\mathrm{Mg}$ & 15.48 & 9.63 & 62 \\
\hline $\mathrm{Si}$ & 5.55 & 4.03 & 73 \\
\hline $\mathrm{Zr}$ & 8.13 & 19.00 & 234 \\
\hline $\mathrm{Al}$ & 0.76 & 0.96 & 127 \\
\hline $\mathrm{K}$ & 0.64 & 0.42 & 65 \\
\hline $\mathrm{Fe}$ & 0.12 & 0.16 & 136 \\
\hline $\mathrm{Zn}$ & 0.02 & 0.03 & 155 \\
\hline $\mathrm{Sc}$ & NA & NA & NA \\
\hline $\mathrm{Sr}$ & 0.01 & 0.01 & 129 \\
\hline $\mathrm{Ti}$ & 0.03 & 0.04 & 119 \\
\hline $\mathrm{Br}$ & 0.00 & 0.00 & 188 \\
\hline $\mathrm{Cr}$ & 0.01 & 0.02 & 127 \\
\hline $\mathrm{Tm}$ & NA & NA & NA \\
\hline $\operatorname{Re}$ & 0.01 & 0.01 & 150 \\
\hline $\mathrm{Fr}$ & 0.00 & 0.00 & 74 \\
\hline $\mathrm{Tb}$ & 0.03 & 0.03 & 123 \\
\hline $\mathrm{Au}$ & NA & NA & NA \\
\hline $\mathrm{Pa}$ & NA & NA & NA \\
\hline Dy & 0.03 & 0.04 & 156 \\
\hline $\mathrm{Mn}$ & NA & NA & NA \\
\hline $\mathrm{Cu}$ & 0.01 & 0.01 & 156 \\
\hline $\mathrm{Ta}$ & NA & NA & NA \\
\hline $\mathrm{Na}$ & 22.44 & 19.06 & 85 \\
\hline $\mathrm{Hf}$ & NA & NA & NA \\
\hline $\mathrm{W}$ & NA & NA & NA \\
\hline $\mathrm{Rb}$ & $\mathrm{NA}$ & $\mathrm{NA}$ & NA \\
\hline $\mathrm{Pt}$ & NA & NA & NA \\
\hline $\mathrm{Sn}$ & NA & NA & NA \\
\hline $\mathrm{Pb}$ & NA & NA & NA \\
\hline $\mathrm{Er}$ & NA & NA & NA \\
\hline $\mathrm{Ni}$ & NA & NA & NA \\
\hline $\mathrm{V}$ & NA & NA & NA \\
\hline Po & NA & NA & NA \\
\hline $\mathrm{Eu}$ & NA & NA & NA \\
\hline $\mathrm{Yb}$ & NA & NA & NA \\
\hline At & NA & NA & NA \\
\hline $\mathrm{Ir}$ & NA & NA & NA \\
\hline $\mathrm{Bi}$ & NA & NA & NA \\
\hline
\end{tabular}


Tables 4 and 5 show the analysis results of different kinds of tap water samples and their statistical parameters, respectively.

Table 4. Percent relative mass concentration of elements contained in tap water samples.

\begin{tabular}{|c|c|c|c|c|c|c|c|c|c|c|}
\hline 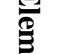 & \multicolumn{10}{|c|}{ Tab water samples } \\
\hline$\%$ & $\mathbf{1}$ & 2 & 3 & 4 & 5 & 6 & 7 & 8 & 9 & 10 \\
\hline $\mathrm{Ca}$ & 41.5 & -- & 34.9 & 24.8 & 82.8 & 81.8 & 67.3 & 33.3 & 17.8 & 17.1 \\
\hline $\mathrm{S}$ & 18.3 & 30.5 & 14.2 & 16.1 & 3.39 & 6.56 & 15.6 & 12.0 & 22.7 & 21.7 \\
\hline $\mathrm{Cl}$ & 15.0 & 48.6 & 13.4 & 28.5 & 3.68 & 0.952 & 3.37 & 7.91 & 19.5 & 28.2 \\
\hline $\mathrm{Mg}$ & 14.6 & 13.6 & 17.4 & 19.0 & 7.32 & 8.90 & 10.6 & 20.1 & 24.8 & 22.8 \\
\hline $\mathrm{Si}$ & 5.57 & 6.35 & 3.97 & 7.56 & 1.92 & 1.40 & 1.75 & 9.42 & 9.46 & 6.43 \\
\hline $\mathrm{Zr}$ & 3.26 & 0.209 & 0.405 & 1.28 & 0.704 & 0.187 & 0.514 & 2.32 & 3.12 & 0.868 \\
\hline $\mathrm{Al}$ & 0.873 & 0.695 & 0.387 & 0.393 & 0.113 & 0.185 & 0.289 & 0.780 & 0.936 & 0.501 \\
\hline $\mathrm{K}$ & 0.284 & --- & 1.39 & 2.10 & 0.023 & --- & 0.391 & 1.44 & 1.14 & 2.31 \\
\hline $\mathrm{Fe}$ & 0.216 & 0.014 & 0.037 & 0.081 & 0.019 & 0.014 & 0.037 & 0.143 & 0.211 & 0.058 \\
\hline $\mathrm{Zn}$ & 0.125 & 0.002 & 0.004 & 0.011 & 0.004 & 0.002 & --- & 0.014 & 0.137 & 0.009 \\
\hline $\mathrm{Sc}$ & 0.056 & --- & --- & --- & --- & --- & 0.042 & --- & 0.077 & --- \\
\hline $\mathrm{Sr}$ & 0.035 & 0.004 & 0.012 & 0.018 & 0.004 & 0.012 & 0.065 & 0.023 & 0.022 & 0.018 \\
\hline $\mathrm{Ti}$ & 0.026 & --- & 0.012 & 0.032 & --- & --- & 0.003 & 0.038 & 0.052 & 0.019 \\
\hline $\mathrm{Br}$ & 0.0137 & 0.0005 & 0.0010 & 0.0020 & 0.0004 & 0.0003 & 0.0026 & 0.0035 & 0.0019 & 0.0015 \\
\hline $\mathrm{Cr}$ & 0.0106 & --- & 0.0022 & 0.0064 & --- & --- & 0.0011 & 0.0113 & 0.0131 & 0.0056 \\
\hline $\mathrm{Tm}$ & 0.0094 & --- & --- & --- & --- & --- & --- & --- & --- & 0.0045 \\
\hline $\mathrm{Re}$ & 0.0064 & 0.0007 & 0.0017 & 0.0022 & --- & 0.0007 & 0.0021 & 0.0024 & 0.0046 & 0.0015 \\
\hline $\mathrm{Fr}$ & 0.0049 & 0.0003 & 0.0015 & 0.0013 & --- & 0.0005 & 0.0022 & 0.0019 & 0.0019 & 0.0012 \\
\hline $\mathrm{Tb}$ & 0.0046 & 0.0021 & --- & 0.0060 & --- & --- & --- & --- & 0.0288 & --- \\
\hline $\mathrm{Au}$ & 0.0039 & 0.0006 & --- & 0.0011 & --- & 0.0004 & 0.0012 & --- & 0.0033 & 0.0017 \\
\hline $\mathrm{Pa}$ & 0.0036 & --- & --- & --- & --- & --- & --- & 0.0029 & --- & --- \\
\hline Dy & --- & 0.0028 & 0.0090 & 0.0173 & --- & 0.0036 & 0.0052 & 0.0038 & 0.0170 & --- \\
\hline $\mathrm{Mn}$ & --- & 0.0021 & --- & --- & --- & --- & --- & 0.0084 & --- & --- \\
\hline $\mathrm{Cu}$ & --- & 0.0009 & 0.0010 & 0.0028 & 0.0007 & 0.0008 & 0.0019 & 0.0032 & 0.0074 & 0.0020 \\
\hline $\mathrm{Ta}$ & --- & 0.0007 & 0.0027 & 0.0018 & --- & --- & 0.0014 & 0.0050 & --- & --- \\
\hline $\mathrm{Na}$ & --- & --- & 13.8 & --- & --- & --- & --- & 12.4 & --- & --- \\
\hline $\mathrm{Hf}$ & --- & --- & 0.0032 & --- & --- & --- & --- & --- & --- & --- \\
\hline $\mathrm{W}$ & --- & --- & 0.0022 & --- & --- & --- & --- & $\begin{array}{l}-- \\
\end{array}$ & --- & --- \\
\hline $\mathrm{Rb}$ & --- & --- & 0.0005 & --- & --- & --- & 0.0005 & --- & --- & --- \\
\hline $\mathrm{Pt}$ & --- & --- & --- & 0.0010 & --- & --- & --- & --- & --- & --- \\
\hline $\mathrm{Sn}$ & --- & --- & --- & --- & 0.0012 & --- & --- & --- & --- & --- \\
\hline $\mathrm{Pb}$ & --- & --- & --- & --- & 0.0002 & --- & --- & --- & --- & --- \\
\hline $\mathrm{Er}$ & --- & --- & --- & --- & --- & --- & 0.0052 & 0.0112 & --- & 0.0045 \\
\hline $\mathrm{Ni}$ & --- & --- & --- & --- & --- & --- & 0.0016 & 0.0020 & --- & 0.0005 \\
\hline $\mathrm{V}$ & --- & --- & --- & --- & --- & --- & 0.0014 & 0.0087 & --- & --- \\
\hline Po & --- & --- & --- & --- & --- & --- & 0.0005 & --- & --- & --- \\
\hline $\mathrm{Eu}$ & --- & --- & --- & --- & --- & --- & --- & 0.0127 & --- & 0.0237 \\
\hline $\mathrm{Yb}$ & --- & --- & --- & --- & --- & --- & --- & 0.0073 & --- & 0.0042 \\
\hline $\mathrm{At}$ & --- & --- & --- & --- & --- & --- & --- & 0.0024 & --- & --- \\
\hline Ir & --- & --- & --- & --- & --- & --- & --- & --- & 0.0043 & --- \\
\hline $\mathrm{Bi}$ & --- & --- & --- & --- & --- & --- & --- & --- & 0.0031 & --- \\
\hline
\end{tabular}

Elements such as $\mathrm{Ca}, \mathrm{Cl}, \mathrm{Mg}, \mathrm{S}, \mathrm{Na}, \mathrm{Si}, \mathrm{Zr}, \mathrm{Al}, \mathrm{K}, \mathrm{Fe}$, and $\mathrm{Zn}$ appear with a high concentration in tap water samples. The element of $\mathrm{Ca}$ were found in all tap water samples with concentration ranging between $0.0 \%$ and $82.8 \%$, a mean value of $44.59 \pm 26.12 \%$ and $\% \mathrm{CV}$ equals to $59 \%$; $\mathrm{Cl}$ with concentration ranging from $0.952 \%$ to $48.6 \%$, a mean value of $16.91 \pm 14.84 \%$ and $\% \mathrm{CV}$ equals to $88 \%, \mathrm{Mg}$ found with concentration ranging from $7.32 \%$ to $24.8 \%$, a mean value of $15.91 \pm 5.91 \%$ and $\% \mathrm{CV}$ equals to $37 \%, \mathrm{~S}$ appears with concentration ranging from $3.39 \%$ to $30.5 \%$, a mean value of $16.11 \pm 7.89 \%$ and $\% \mathrm{CV}$ equals to $49 \%$; $\mathrm{Na}$ found with concentration ranging between $0.0 \%$ to $13.8 \%$, a mean and $\% \mathrm{CV}$ of negligible value; Si found with concentration between $1.40 \%$ to $9.46 \%$, a mean of $5.38 \pm 3.03$ and a $\% \mathrm{CV}$ equal to $56 \%$; $\mathrm{Zr}$ found with concentration ranging from $0.187 \%$ to $3.26 \%$, a mean value of 
$1.29 \pm 1.18 \%$ and $\% \mathrm{CV}$ equals to $92 \%$; $\mathrm{Al}$ found with concentration ranging between $0.113 \%$ to $0.936 \%$, a mean of $0.52 \pm 0.29 \%$ and $\% \mathrm{CV}$ equal to $56 \%$; $\mathrm{K}$ found with concentration ranging between $0.0 \%$ to $2.31 \%$, a mean value of $1.13 \pm 0.84 \%$ and $\% \mathrm{CV}$ equal to $74 \%$, Fe found with concentration ranging between $0.014 \%$ to $0.216 \%$, a mean value of $0.08 \pm 0.08$ and $\% \mathrm{CV}$ of $95 \%$ and $\mathrm{Zn}$ found with concentration ranging between $0.0 \%$ to $0.137 \%$, a mean value of $0.03 \pm 0.06$ and $\% \mathrm{CV}$ of $161 \%$.

Table 5. Statistical parameters for elements contained in tap water samples.

\begin{tabular}{|c|c|c|c|}
\hline$\%$ Elements & $\%$ Mean & SD & $\% \mathrm{CV}$ \\
\hline $\mathrm{Ca}$ & 44.59 & 26.12 & 59 \\
\hline $\mathrm{S}$ & 16.11 & 7.89 & 49 \\
\hline $\mathrm{Cl}$ & 16.91 & 14.84 & 88 \\
\hline $\mathrm{Mg}$ & 15.91 & 5.91 & 37 \\
\hline $\mathrm{Si}$ & 5.38 & 3.03 & 56 \\
\hline $\mathrm{Zr}$ & 1.29 & 1.18 & 92 \\
\hline $\mathrm{Al}$ & 0.52 & 0.29 & 56 \\
\hline $\mathrm{K}$ & 1.13 & 0.84 & 74 \\
\hline $\mathrm{Fe}$ & 0.08 & 0.08 & 95 \\
\hline $\mathrm{Zn}$ & 0.03 & 0.06 & 161 \\
\hline Sc & NA & NA & NA \\
\hline $\mathrm{Sr}$ & 0.02 & 0.02 & 84 \\
\hline $\mathrm{Ti}$ & 0.03 & 0.02 & 63 \\
\hline $\mathrm{Br}$ & 0.00 & 0.00 & 145 \\
\hline $\mathrm{Cr}$ & 0.01 & 0.00 & 64 \\
\hline $\mathrm{Tm}$ & NA & NA & NA \\
\hline $\mathrm{Re}$ & 0.00 & 0.00 & 75 \\
\hline $\mathrm{Fr}$ & 0.00 & 0.00 & 77 \\
\hline $\mathrm{Tb}$ & NA & NA & NA \\
\hline $\mathrm{Au}$ & 0.00 & 0.00 & 77 \\
\hline $\mathrm{Pa}$ & NA & NA & NA \\
\hline Dy & 0.01 & 0.01 & 75 \\
\hline $\mathrm{Mn}$ & NA & NA & NA \\
\hline $\mathrm{Cu}$ & 0.00 & 0.00 & 92 \\
\hline $\mathrm{Ta}$ & 0.00 & 0.00 & 72 \\
\hline $\mathrm{Na}$ & NA & NA & NA \\
\hline Hf & NA & NA & NA \\
\hline $\mathrm{W}$ & NA & NA & NA \\
\hline $\mathrm{Rb}$ & NA & NA & NA \\
\hline $\mathrm{Pt}$ & NA & NA & NA \\
\hline $\mathrm{Sn}$ & NA & NA & NA \\
\hline $\mathrm{Pb}$ & NA & NA & NA \\
\hline $\mathrm{Er}$ & NA & NA & NA \\
\hline $\mathrm{Ni}$ & NA & NA & NA \\
\hline $\mathrm{V}$ & NA & NA & NA \\
\hline Po & NA & NA & NA \\
\hline $\mathrm{Eu}$ & NA & NA & NA \\
\hline $\mathrm{Yb}$ & NA & NA & NA \\
\hline At & NA & NA & NA \\
\hline $\mathrm{Ir}$ & NA & NA & NA \\
\hline $\mathrm{Bi}$ & NA & NA & NA \\
\hline
\end{tabular}

Tables 6 and 7 show elements are containing a high concentration in $(\mathrm{mg} / \mathrm{L})$ in bottle and tab water. Table 8 shows the concentration range for metal and nonmetal elements detected by the XRF technique in bottles and tap water compared with the WHO international standard [15].

All elements contained in Table 8 are found within the accepted level of the international standard level of the WHO except for $\mathrm{Ca}, \mathrm{Al}, \mathrm{Mg}$, and $\mathrm{Cr}$, and it is named "Risky elements" since they are involved in the number of series of diseases. An element of Ca and $\mathrm{Al}$ in the bottle and the tap water exceeds the WHO limits by 3.6 to 10 and 19 to 53 times, 
respectively. The element of $\mathrm{Mg}$ and $\mathrm{Cr}$ in only tab water exceeds the WHO limits by 1.4 and

2.6 times, respectively.

Table 6. Elements contained in bottled water in $\mathrm{mg} / \mathrm{L}$.

\begin{tabular}{|c|c|c|c|c|c|c|c|c|c|c|c|c|c|c|c|}
\hline Sample No. & $\mathrm{Ca}$ & $\mathbf{S}$ & $\mathrm{Cl}$ & Mg & Si & $\mathrm{Zr}$ & Al & $\mathbf{K}$ & $\mathrm{Fe}$ & $\mathbf{Z n}$ & $\mathrm{Na}$ & $\mathrm{Cr}$ & $\mathrm{Cu}$ & $\mathbf{P b}$ & $\mathbf{N i}$ \\
\hline 1 & 0.000 & 0.853 & 1.068 & 35.40 & 3.270 & 87.15 & 0.000 & 0.000 & 0.027 & 0.007 & 22.20 & 0.0000 & 0.0006 & 0.000 & 0.000 \\
\hline 2 & 4.325 & 8.587 & 14.75 & 10.05 & 10.30 & 0.920 & 0.710 & 0.427 & 0.111 & 0.017 & 74.75 & 0.0050 & 0.0046 & 0.000 & 0.000 \\
\hline 3 & 126.2 & 0.933 & 1.195 & 13.33 & 2.100 & 0.210 & 0.077 & 0.000 & 1.470 & 0.180 & 5.820 & 0.0000 & 0.0000 & 0.000 & 0.000 \\
\hline 4 & 179.5 & 10.59 & 7.482 & 19.87 & 3.420 & 0.000 & 0.292 & 0.000 & 0.000 & 0.000 & 16.34 & 0.0000 & 0.0000 & 0.000 & 0.000 \\
\hline 5 & 147.5 & 10.74 & 1.608 & 12.51 & 1.723 & 0.404 & 0.353 & 0.000 & 0.025 & 0.001 & 0.000 & 0.0000 & 0.0000 & 0.000 & 0.000 \\
\hline 6 & 21.40 & 21.60 & 18.76 & 54.00 & 20.00 & 1.300 & 1.002 & 2.660 & 0.083 & 0.004 & 58.40 & 0.0118 & 0.0022 & 0.000 & 0.003 \\
\hline 7 & 33.67 & 5.010 & 3.300 & 15.30 & 6.607 & 7.500 & 2.242 & 0.498 & 0.356 & 0.044 & 0.000 & 0.0484 & 0.0363 & 0.000 & 0.000 \\
\hline 8 & 0.000 & 55.30 & 53.20 & 7.385 & 10.11 & 0.021 & 0.000 & 0.000 & 0.000 & 0.000 & 49.00 & 0.0000 & 0.0000 & 0.000 & 0.000 \\
\hline 9 & 70.20 & 9.457 & 32.01 & 32.01 & 24.86 & 19.01 & 3.867 & 0.864 & 0.950 & 0.364 & 0.122 & 0.0217 & 0.0000 & 0.000 & 0.000 \\
\hline 10 & 3.357 & 84.36 & 11.49 & 67.57 & 9.732 & 1.999 & 2.358 & 0.603 & 0.146 & 0.012 & 30.81 & 0.0085 & 0.0042 & 0.000 & 0.000 \\
\hline
\end{tabular}

Table 7. Elements contained in tap water samples in $\mathrm{mg} / \mathrm{L}$.

\begin{tabular}{|c|c|c|c|c|c|c|c|c|c|c|c|c|c|c|c|}
\hline Sample No. & $\mathrm{Ca}$ & $\mathbf{S}$ & Cl & Mg & Si & $\mathbf{Z r}$ & Al & $\mathbf{K}$ & $\mathrm{Fe}$ & $\mathrm{Zn}$ & $\mathrm{Na}$ & $\mathrm{Cr}$ & $\mathrm{Cu}$ & $\mathbf{P b}$ & $\mathbf{N i}$ \\
\hline 1 & 508.4 & 224.2 & 183.7 & 178.8 & 68.23 & 39.93 & 10.69 & 3.479 & 2.646 & 1.531 & 0 & 0.129 & 0 & 0 & 0 \\
\hline 2 & 0 & 80.06 & 127.5 & 35.7 & 16.66 & 0.548 & 1.824 & 0 & 0.037 & 0.005 & 0 & 0 & 0.002 & 0 & 0 \\
\hline 3 & 87.25 & 35.5 & 33.5 & 43.5 & 9.925 & 1.013 & 0.967 & 3.475 & 0.093 & 0.01 & 34.5 & 0.005 & 0.005 & 0 & 0 \\
\hline 4 & 49.6 & 32.2 & 57 & 38 & 15.12 & 2.56 & 0.786 & 4.2 & 0.162 & 0.022 & 0 & 0.012 & 0.005 & 0 & 0 \\
\hline 5 & 155.3 & 6.356 & 6.9 & 13.72 & 3 & 1.32 & 0.212 & 0.043 & 0.036 & 0.007 & 0 & 0 & 0.001 & 0 & 0 \\
\hline 6 & 296.5 & 23.78 & 3.451 & 32.26 & 5.075 & 0.677 & 0 & 0.051 & 0.007 & 0 & 0.043 & 0 & 0.003 & 0 & 0 \\
\hline 7 & 487.9 & 113.1 & 24.43 & 76.85 & 12.68 & 3.726 & 2.095 & 2.835 & 0.268 & 0 & 0 & 0.008 & 0.014 & 0 & 0.01 \\
\hline 8 & 79.08 & 28.5 & 18.78 & 47.73 & 22.37 & 5.51 & 1.852 & 3.42 & 0.339 & 0.033 & 29.45 & 0.027 & 0.008 & 0 & 0.021 \\
\hline 9 & 84.55 & 107.8 & 92.62 & 117.8 & 44.93 & 14.82 & 4.446 & 5.415 & 1.002 & 0.65 & 0 & 0.062 & 0.035 & 0 & 0 \\
\hline 10 & 38.47 & 48.82 & 63.45 & 51.3 & 14.46 & 1.953 & 1.127 & 5.197 & 0.13 & 0.02 & 0 & 0.013 & 0.005 & 0 & 0.001 \\
\hline
\end{tabular}

There is a significant inverse relationship between the levels of Calcium and Magnesium in drinking water and the risk of death from breast cancer, kidney stones, gastric cancer, and the risk of cerebrovascular disease [16-18]. Also, a positive relationship between the increase in aluminum level in drinking water and Alzheimer's disease was indicated [19]. Chromium enters the air, water, and soil mostly in the Chromium (III) and $\mathrm{Cr}$ (VI) forms. $\mathrm{Cr}$ (III) is an essential nutrient that helps the body use sugar, protein, and fat.

Table 8. Elements concentration in $(\mathrm{mg} / \mathrm{L})$ detected by the XRF technique compared to the WHO standard for bottled and tap water samples.

\begin{tabular}{|c|c|c|c|}
\hline \multirow{3}{*}{ Elements } & \multirow{3}{*}{$\begin{array}{l}\text { WHO Standard(mg/L) } \\
(\mathbf{2 0 1 1})\end{array}$} & \multicolumn{2}{|c|}{ XRF Technique } \\
\hline & & Bottled Water & Tab Water \\
\hline & & $(\mathrm{mg} / \mathrm{L})$ & $(\mathrm{mg} / \mathrm{L})$ \\
\hline $\mathrm{Cl}$ & 250 & $1.06-53.2$ & $6.90-183.7$ \\
\hline $\mathrm{F}$ & 1.5 & NA & NA \\
\hline $\mathrm{Ar}$ & 0.01 & NA & NA \\
\hline $\mathrm{B}$ & 10 & NA & NA \\
\hline $\mathrm{Na}$ & 200 & $0.00-74.7$ & $0.00-34.5$ \\
\hline $\mathrm{Ca}$ & $25.0-50.0$ & $0.000-179.5$ & $0.000-508.3$ \\
\hline $\mathrm{Al}$ & $0.10-0.20$ & $0.000-3.867$ & $0.000-10.69$ \\
\hline $\mathrm{K}$ & NA & $0.000-2.660$ & $0.000-5.410$ \\
\hline $\mathrm{Zr}$ & NA & $0.000-87.15$ & $0.540-39.90$ \\
\hline $\mathrm{Mg}$ & $50-125$ & $7.380-67.57$ & $13.70-178.8$ \\
\hline $\mathrm{Cd}$ & 0.003 & NA & NA \\
\hline $\mathrm{Cr}$ & 0.05 & $0.000-0.048$ & $0.000-0.129$ \\
\hline $\mathrm{Cu}$ & 2 & $0.000-0.036$ & $0.000-0.035$ \\
\hline $\mathrm{Pb}$ & 0.01 & 0 & 0 \\
\hline $\mathrm{Hg}$ & 0.006 & NA & NA \\
\hline $\mathrm{Ni}$ & 0.07 & 0 & $0.000-0.003$ \\
\hline $\mathrm{U}$ & 0.03 & 0 & 0 \\
\hline
\end{tabular}

NA: Not available 
Breathing high levels of chromium (VI) can irritate the nose, such as the runny nose, nosebleeds, ulcers, and holes in the nasal septum. Ingesting large amounts of Chromium (VI) can cause stomach upsets and ulcers, convulsions, kidney and liver damage, and cancerous growths.

In this study, the selected statistical parameters emphasize the significance of water intake on the risk of developing the disease and to make it possible to predict the relationships.

When the mean value is low, large SD results in a large \% CV; this indicates that the risk of developing a certain disease is high. In contrast, if both the mean and SD are high but with a low $\% \mathrm{CV}$, the disease's risk will be relatively low. A comparable study can be made for the formation of kidney stone diseases, where the high mean of $\% \mathrm{CV}$ is relatively insensitive to the magnitude value of the SD and will lead to a high risk of developing a disease. Table 9 shows the statistical parameters and type of risk to develop diseases for the four risky elements.

Table 9. Risky elements, statistical parameters, and degree of risk.

\begin{tabular}{|c|c|c|c|c|}
\hline \multirow[b]{2}{*}{ Elements } & & & & \multirow{4}{*}{$\begin{array}{l}\text { Degree of risk } \\
\text { High Risk }\end{array}$} \\
\hline & Source of water & Mean \pm SD & $\% \mathrm{CV}$ & \\
\hline \multirow[t]{2}{*}{$\mathrm{Ca}$} & Bottle & $43.49 \pm 35.44$ & 81 & \\
\hline & $\mathrm{Tab}$ & $44.12 \pm 26.12$ & 59 & \\
\hline \multirow{2}{*}{$\mathrm{Al}$} & Bottle & $0.76 \pm 0.96$ & 127 & \multirow{2}{*}{ High Risk } \\
\hline & $\mathrm{Tab}$ & $0.52 \pm 0.29$ & 56 & \\
\hline \multirow[t]{2}{*}{$\mathrm{Mg}$} & Bottle & $15.48 \pm 9.63$ & 62 & \multirow[t]{2}{*}{ Low Risk } \\
\hline & $\mathrm{Tab}$ & $15.91 \pm 5.91$ & 37 & \\
\hline \multirow[t]{2}{*}{$\mathrm{Cr}$} & Bottle & $0.01 \pm 0.02$ & 127 & \multirow[t]{2}{*}{ High Risk } \\
\hline & $\mathrm{Tab}$ & $0.01 \pm 0.00$ & 64 & \\
\hline
\end{tabular}

The statistical parameters for the four risky elements in Table 9 show a high mean value for the element of $\mathrm{Ca}$ in bottle and tab water. The \% $\mathrm{CV}$ is relatively insensitive to the magnitude value of the SD, which leads to a high risk of developing diseases, especially when genetic factors are excluded. The statistical parameters for aluminum in bottle and tab water indicate a low mean value with high $\mathrm{SD}$ and high \% $\mathrm{CV}$, which leads to a high risk of developing diseases. Additionally, the statistical parameter for $\mathrm{Mg}$ in bottle and tab water indicates a high mean and SD for which the $\% \mathrm{CV}$ is low, which leads to a low risk of developing diseases. Finally, the statistical parameter for $\mathrm{Cr}$ in bottle and tab water indicates a low mean and high SD for which the \% CV is high, which leads to a high risk of developing diseases.

\section{Conclusions}

The four elements $\mathrm{Ca}, \mathrm{Al}, \mathrm{Mg}$, and $\mathrm{Cr}$ were identified as risky elements for the formation of diseases in the drinking water of Koya town. $\mathrm{Ca}, \mathrm{Al}$, and $\mathrm{Cr}$ levels exceeded the WHO standard and were identified as high risky elements. According to the statistical parameter analysis, Mg level exceeds that for the WHO limits but is identified with low risk in developing diseases.

Although water consumption containing such elements may elevate the risk of developing diseases, these elements are also essential for the human body to function within the accepted level. The element composition in the water becomes increasingly important to understand their impact on human health; however, genetic factors should always be considered, which indicates the need for extensive work in the future. 


\section{Funding}

This research received no external funding.

\section{Acknowledgments}

The authors gratefully acknowledge the staff at Koya hospital and the Physics Department of Koya University for their kind assistance in providing and analyzing the samples in this study.

\section{Conflicts of Interest}

The authors declare no conflict of interest.

\section{References}

1. Anthonj, C.; Diekkrüger, B.; Borgemeister, C.; Thomas, K. Health risk perceptions and local knowledge of water-related infectious disease exposure among Kenyan wetland communities. International Journal of Hygiene and Environmental Health 2019, 222, 34-48, https://doi.org/10.1016/j.ijheh.2018.08.003.

2. Kamarehie, B.; Jafari, A.; Zarei, A.; Fakhri, Y.; Ghaderpoori, M.; Alinejad, A. Non-carcinogenic health risk assessment of nitrate in bottled drinking waters sold in Iranian markets: A Monte Carlo simulation. Accreditation and Quality Assurance 2019, 24, 417-426, https://doi.org/10.1007/s00769-019-01397-5.

3. Semerjian, L.; Alrajaby, H.; Naaz, N.; Kasfah, R.; Dalah, E.Z.; Waheed, E.; Nabulssi, A.; Metwally, W.A. Age-dependent effective ingestion dose estimations and lifetime risk assessment for selected radionuclides $(40 \mathrm{~K}$ and $3 \mathrm{H})$ in bottled waters marketed in United Arab Emirates. Chemosphere 2020, 249, 1 https://doi.org/10.1016/j.chemosphere.2020.126114.

4. Joseph, N.; Bhat, S.; Mahapatra, S.; Singh, A.; Jain, S.; Unissa, A.; Janardhanan, N. Bacteriological Assessment of Bottled Drinking Water Available at Major Transit Places in Mangalore City of South India. Journal of Environmental and Public Health 2018, 2018, https://doi.org/10.1155/2018/7472097.

5. Gao, B.; Gao, L.; Gao, J.; Xu, D.; Wang, Q.; Sun, K. Simultaneous evaluations of occurrence and probabilistic human health risk associated with trace elements in typical drinking water sources from major river basins in China. Science of The Total Environment 2019, 666, 139-146, https://doi.org/10.1016/j.scitotenv.2019.02.148.

6. Nakano, T.; Yamashita, K.; Ando, A.; Kusaka, S.; Saitoh, Y. Geographic variation of Sr and S isotope ratios in bottled waters in Japan and sources of $\mathrm{Sr}$ and S. Science of The Total Environment 2020, 704, https://doi.org/10.1016/j.scitotenv.2019.135449.

7. Barrington, D.J.; Sridharan, S.; Saunders, S.G.; Souter, R.T.; Bartram, J.; Shields, K.F.; Meo, S.; Kearton, A.; Hughes, R.K. Improving community health through marketing exchanges: A participatory action research study on water, sanitation, and hygiene in three Melanesian countries. Social Science \& Medicine 2016, 171, 84-93, https://doi.org/10.1016/j.socscimed.2016.11.003.

8. Nnorom, I.C.; Ewuzie, U.; Eze, S.O. Multivariate statistical approach and water quality assessment of natural springs and other drinking water sources in Southeastern Nigeria. Heliyon 2019, 5, https://doi.org/10.1016/j.heliyon.2019.e01123.

9. Lugo, J.; Lugo Arias, E.; Puente, M. A systematic review of microorganisms as indicators of recreational water quality in natural and drinking water systems. Journal of Water and Health 2020, 19, 20-28, https://doi.org/10.2166/wh.2020.179.

10. Fareed, S.J.S.M.I. Evaluation of Drinking Water Quality in Erbil City Kurdistan, Region-Iraq. Evaluation 2015, 5, https://doi.org/10.7176/JEES.

11. Jadoon, S.; Munir, S.; Abdulhamid, A.M. Analyzing Water Quality in Darbandikhan Reservoir, Kurdistan Region, Iraq. Environmental Science 2016, https://doi.org/10.21275/v4i12.sub158579.

12. Mohammed Barzinji D.A.; Ganjo, D.G.A. Assessment of the Chemical Quality of Water in the HalabjaSulaimani, Kurdistan Region of Iraq. Asian Journal of Water, Environment and Pollution 2014, 11, 19-28.

13. Kafia, M.S.; Shareef; Slaiman, G.M.; Muhamad, S.; Nazanin, M.S.; Shekhani. Physical and Chemical Status of Drinking Water from Water Treatment Plants on Greater Zab River. Journal of Applied Sciences and Environmental Management 2009, 13, 89-92, https://doi.org/10.4314/jasem.v13i3.55371.

14. Wählisch, A.; Streeck, C.; Hönicke, P.; Beckhoff, B. Validation of secondary fluorescence excitation in quantitative X-ray fluorescence analysis of thin alloy films. Journal of Analytical Atomic Spectrometry 2020, 35, 1664-1670, https://doi.org/10.1039/D0JA00171F.

15. Li, P.; Wu, J. Drinking Water Quality and Public Health. Exposure and Health 2019, 11, 73-79, https://doi.org/10.1007/s12403-019-00299-8. 
16. Kozisek, F. Regulations for calcium, magnesium or hardness in drinking water in the European Union member states. Regulatory Toxicology and Pharmacology 2020, 112, https://doi.org/10.1016/j.yrtph.2020.104589.

17. Hailu, Y.; Tilahun, E.; Brhane, A.; Resky, H.; Sahu, O. Ion exchanges process for calcium, magnesium and total hardness from ground water with natural zeolite. Groundwater for Sustainable Development 2019, 8, 457-467, https://doi.org/10.1016/j.gsd.2019.01.009.

18. Jensen, A.C.S.; Imberti, S.; Habraken, W.J.E.M.; Bertinetti, L. Small Ionic Radius Limits Magnesium Water Interaction in Amorphous Calcium/Magnesium Carbonates. The Journal of Physical Chemistry C 2020, 124, 6141-6144, https://doi.org/10.1021/acs.jpcc.9b11594.

19. Van Dyke, N.; Yenugadhati, N.; Birkett, N.J.; Lindsay, J.; Turner, M.C.; Willhite, C.C.; Krewski, D. Association between aluminum in drinking water and incident Alzheimer's disease in the Canadian Study of Health and Aging cohort. NeuroToxicology 2021, 83, 157-165, https://doi.org/10.1016/j.neuro.2020.04.002. 